\title{
Repurposing Cardio-Metabolic Drugs to Fight Covid19
}

\author{
Allegra Battistoni $^{1}\left[\right.$ [Dassimo Volpe ${ }^{1}[$ \\ Received: 28 August 2021 / Accepted: 3 September 2021 / Published online: 15 September 2021 \\ (c) Italian Society of Hypertension 2021
}

Keywords Repurposing $\cdot$ Drugs $\cdot$ Covid19 $\cdot$ Statins

Since November 2019, healthcare systems around the world had to face the consequences of the pandemic caused by the Sars-CoV-2 virus. This virus is a positive-sense singlestranded RNA virus classified as coronavirus. Two previous epidemics caused by coronaviruses developed in 2000s, but had been confined to specific regions of the world. On the contrary, the disease associated with Sars-Cov-2 infection, known as Covid19, due to its very high contagiousness, has taken on the measure of a pandemic. To date, more than two hundred million cases are confirmed, with over four million deaths globally [1].

Covid19 mostly manifests with interstitial pneumonia, even though it can affect heart and central nervous system as well. Although the pathophysiology of the clinical manifestations of Covid19 is not yet completely known, it has been shown that the entry of Sars-CoV-2 into cells is mediated by angiotensin-converting enzyme 2 (ACE2), expressed also in pulmonary epithelium [2]. After that, symptoms and the severity of Covid19 depend on the virus replication but also on the individual's immune response, often establishing an immune/inflammatory storm leading to tissue damage that can have severe prognosis [2, 3].

In parallel with the progressive knowledge about the mechanisms of Sars-CoV-2 infection and the pathophysiology of Covid19, the search for possible therapeutic targets for drugs aimed at treating patients affected by Covid 19 has been developed. However, the design of new drugs requires time and investments. Indeed, the cost of a new drug is estimated to more than a billion dollars extending for a period of 10-15 years [4] with a success rate of only $2.01 \%$ [5]. For this reason, most of the efforts have been lately used

Allegra Battistoni

allegra.battistoni@uniroma1.it

1 Department of Clinical and Molecular Medicine, University of Rome Sapienza, Sant'Andrea Hospital, Via di Grottarossa 1035, 00189 Rome, Italy in a titanic way to develop vaccines to stem the spread of the virus and hopefully eradicate it. Currently four vaccines (Tozinameran/BNT162b2/Comirnaty by Pfizer/BioNTech), mRNA-1273 by Moderna), AZD1222/Covishield by University of Oxford/AstraZeneca and Ad26.COV2.S/JNJ78436735 by Janssen Vaccines and Prevention) have been developed in western countries and more than 5 billion doses have already been administered worldwide [1] .

However, the search for drugs useful in treating Covid19 in people already affected is not of secondary importance. This is particularly crucial if we consider the likely development and spread of new variants of the virus, the percentage of people not able to be immunized globally due to the presence of comorbidities and the evidence that currently only $33 \%$ of the world population have received at least one doses. In particular, populous countries with the lower incomes, such as India, do not have free access to vaccines, with only $1.6 \%$ of people in low-income countries have received at least one dose [6].

Although several drugs are under active investigation, there are currently no effective drugs against SARS-CoV-2 infection [7]. The most used therapeutic schemes consist of glucocorticoids and anticoagulants as the immune storm as well as the damage of the endothelium and thrombotic processes seem critical in the evolution of the infection and prognosis. Data from the large RECOVERY trial, a controlled open-label trial involving more than 6000 patients, have shown that in patients hospitalized with Covid19, the use of dexamethasone resulted in lower 28-day mortality among those patients on respiratory support [8].

Since the beginning of the pandemic, the repurpose of drugs, which is the process of identifying new uses for approved drugs, has been suggested for the treatment of Covid19, due to the enormous need for health sources and high economic burden of the pandemic. Based on viral pathogenesis and pharmacodynamics, many agents might be repurposed [9]. Drug repurposing is based on two principles. 
The first is that a single drug interacts with multiple targets, so that a drug already known might have new targets of action $[10,11]$. The second is that targets associated with a disease might be involved in many biological processes $[12,13]$ so that a known target might have a new indication. Therefore, it has been considered that $75 \%$ of known drugs could be repositioned for various diseases [9]. Therefore, during the first year of the pandemic, several drugs were repurposed including those drugs acting through virus related targets such as RNA genome (i.e. remdesivir); drugs acting through polypeptide packing (i.e. lopinavir and ritonavir); drugs acting through host targets such as antiviral immunity (i.e. interferons), drugs targeting the virus uptake pathways (i.e. Chloroquine) and drugs acting on host proinflammatory cytokines (i.e. Tocilizumab) [14]. Few of these drugs have been also tested in the Solidarity clinical trial for Covid19 [15], which is a multinational phase III-IV clinical trial organized by the World Health Organization (WHO) and partners to compare four untested treatments for hospitalized people with severe Covid19 illness.

Traditionally, the repurposing of an existing agent, appealing due to its biological plausibility, takes much experimental work both in vitro and in vivo. Expertise in pharmacology, as well as expertise in translational drug development and clinical trials is needed to set up smallscale studies and then larger population-wide study are crucial to confirm the use of a given compound for a new indication [16]. Although large randomized trials have begun to identify drugs that can be repurposed to face Covid19 [17-21], many of them have been conducted only in hospitalized and severely ill patients mostly failing to show any efficacy. Therefore, to identify additional drugs that can be repurposed also for early management in Covid19, remains a priority.

Alongside with this "traditional" process based on pathophysiology and pharmacodynamics, systems available in the 21 st century have made it possible to add other ways to identify drugs already on the market and potentially useful to treat Covid19. Broadly, there are three kinds of approaches that can be used in drug repositioning: biological experimental approaches (the traditional ones), computational approaches and mixed approaches that can be especially useful in emergency situations such as the Covid19 pandemic [14].

Many computational methods are now available to examine the relationships between chemical compounds, molecular targets, biological systems, therapeutic endpoints and diseases [22]. For example, it has been presented a new network-based algorithm for drug repositioning, called SAveRUNNER (Searching off-lAbel dRUg aNd NEtwoRk), which predicts drug-disease associations by quantifying the interplay between the drug targets and the disease-specific proteins in the human interactome via a novel network-based similarity measure that detect associations between drugs and diseases locating in the same network neighborhoods. With this approach, many drugs possibly useful in Covid 19 have been found including ACE-inhibitors (ACEi), monoclonal antibodies (e.g., anti-IFN $\gamma$, anti-TNF $\alpha$, anti-IL12, anti-IL1 $\beta$, anti-IL6), and thrombin inhibitors [23].

Moreover, another study by Fehr et al. [24] revealed that SARS-CoV-2 infects human cells by using the host's translation pathway to produce twenty-nine viral proteins. These twenty-nine proteins bind to multiple human proteins to set up the molecular processes necessary to develop the infection. Among these protein, Gorden et al. [25] using affinity purification mass spectrometry released the twentysix proteins that the SARS-CoV-2 uses to infect the human body and identified more than three hundred human proteins involved in these viral proteins binds. More than sixty of these proteins appear to be druggable human proteins with already existing drugs. Therefore, the identification of interactions between host and virus proteins might provide significant insights to find drug targets for drug development against SARS-CoV-2.

Among the new approaches for drug repurposing, Mendelian randomization (MR) analyses have also been used. MR analysis focusing on actionable genes, defined as genes that encode proteins targets of drugs already existent, could therefore contribute to repurpose drugs useful in the treatment of Covid19 [21].

One of the most important studies in repurposing of drugs against Covid 19 have been conducted by a team from Ohio, USA [26]. In this study, based on bioinformatics analysis and generated networks of functional drug-gene interactions, the authors identified 16 substances, with different wellknown primary indications and with concurrent anti-SarsCov-2 properties such as irbesartan, toremifene, camphor, equilin, mesalazine, mercaptopurine, paroxetine, sirolimus, carvedilol, colchicine, dactinomycin, melatonin, quinacrine, eplerenon, emodin and oxymetholone.

At last, mostly based on hypotheses derived from observational studies on large cases in which patients taking specific drugs seemed to have a different prognosis, other drugs have been proposed. With this regard, great interest has risen with respect to the potential beneficial effects against Covid19 of drugs that are currently used for cardiovascular (CV) prevention [27, 28]. Indeed, although the main clinical manifestations of Covid19 involve the respiratory system, an increased risk of CV diseases, namely myocarditis, arrhythmias, and coronary artery disease, has been reported in Covid19 patients [29-31]. Moreover, underlying CVD and $\mathrm{CV}$ risk factors have been associated with worse prognosis in Covid19 patients [32].

With this regard, statins appeared to be particularly promising. Not only they lower cholesterol levels acting as 3-hydroxy-3-methylglutaryl coenzyme A reductase 
inhibitors, but they also play many pleiotropic effects. They can reduce vascular and endothelial inflammation, improve endothelial function also by ameliorating the vascular redox balance and inhibit platelet aggregation [33] Moreover, they seem to have an immunomodulatory activity by modifying the release of cytokines during the cytokine storm and the expression of major histocompatibility complex class II increasing the production of protective Th-2 cytokines [34]. Moreover, statins can exert some direct antiviral activity by inhibiting cholesterol synthesis, which is involved in the viral cell cycle [35]. Indeed, the presence of cholesterol-rich subdomains on the plasma membrane of host cells, namely lipid rafts, is crucial for viral fusion and entry [36, 37] In addiction, via epigenetic histone modifications, statins might upregulate the expression of ACE2 [38] which is known to be associated with reduced severity of acute respiratory distress syndrome (ARDS) [39].

Last year, Zhang et al., reported the first large observational study of statin use in Covid19 [40]. In a retrospective cohort 13,981 patients hospitalized with Covid19 in Hubei Province, China, among which 1219 received statins they examined the association between statin use and outcomes. The authors found that the risk for 28- day all-cause mortality was $5.2 \%$ in the statin group and $9.4 \%$ in the matched non-statin group (HR 0.58; 95\% CI, 0.43-0.80; $\mathrm{p}=0.001$ ). Despite the promising results, it must be stated that there are important limitations in this study, such as its retrospective nature, the lack of data on pre-hospital statin use and concomitant therapies, suboptimal matching techniques between the two groups. To date, even though consistent evidence from prospective studies is not currently available, most of available data from retrospective studies support the beneficial effect of statins against SARS-CoV-2 infection in terms of inflammatory parameters, severity of clinical manifestations and mortality [41]. On the contrary Cariou et al. [42], using data from the large-scale multicenter CORONADO study carried out in France in diabetic patients hospitalized for Covid19, reported that routine statin treatment is significantly associated with increased mortality (based on 7-day and 28-day in-hospital death rates).

Recently, a total of eleven highly heterogeneous observational studies were included in a meta-analysis for a total of 3462 statin users and 10,560 non-users. Although no significant reductions in either in-hospital mortality or Covid19 severity were reported among statin users compared with non-users after univariate comparisons, such reductions were observed after adjusting for confounding factors [43].

The most recent available meta-analysis [44] was limited to seven retrospective studies performed in Western countries with 2398 patients with $44.8 \%$ taking statins. Five out of seven studies evaluated patients with statin treatment before hospital admission while the others considered both treatments started before admission or during hospital stay.
Covid19 patients taking statins had nearly $40 \%$ lower odds of incur in the composite endpoint of severe illness or death (OR: 0.59 ; 95\% CI: 0.35-0.99). with only one study [45] reporting an OR > 1 (i.e., OR: 1.60; 95\% CI: 0.88-2.92).

Lately, it has been also proposed that fenofibrates, suppressing inflammation and apoptosis cascades through inactivation of NF-kB and stimulation of adenosine monophosphate-activated protein kinase (AMPK) signaling, might be useful in treating Covid19 [46].

Moreover, metformin, which has been firstly discovered in the search for antimalarial agents and now has become one of the most prescribed antidiabetic drugs worldwide seems to have a new potential therapeutic application in Covid19 [47]. Indeed, retrospective studies [48] and meta-analyses reported significant reduction of infection-related mortality in patients affected by Covid 19 and treated with metformin $[49,50]$. Currently, the "COVIDOUT-Outpatient Treatment of COVID-19 with Metformin" (REGISTRATION: URL: https://www.clinicaltrials.gov NCT04510194) a phase II/III trial involving 750 participants aged $30-85$ years is investigating whether metformin treatment in non-hospitalized adults with SARS-CoV-2 can (a) prevent hypoxia and emergency department utilization, (b) prevent disease progression in Covid19, and (c) improve viral load and C-reactive protein (CRP). Moreover, a phase II trial "Pilot Study into the Use of Metformin and Low Dose Naltrexone (LDN) for Patients with Coronavirus Disease 2019 (COVID-19)-Assessment of Short and Long Term Effects" (REGISTRATION: URL: https://www.clinicaltrials.gov; NCT04604678) involving 80 participants aged $30-70$ years aims to study monthly the effect of a combination of metformin and LDN on hard and soft outcomes in Covid19 patients.

Furthermore, through an integrative bioinformatics approach including the search of the biomedical literature for high confidence DPP4-protein/gene associations followed by functional analysis using network analysis and pathway enrichment it has been found that DPP4 networks are highly enriched in viral processes required for viral entry and infection, and as a result, DPP4 was proposed as an important possible target for the treatment of Covid19. Since protein-chemical interaction networks identified strong interactions between DPP4 and sitagliptin, this latter has been postulated to be beneficial for the treatment of Covid19 disease, either as monotherapy or in combination with other therapies, especially for high CV risk patients [51].

Finally, since Covid19 is associated with the ARDS and higher activity of ACE2 leads to attenuation of ARDS and people taking angiotensin receptor blockers (ARBs) or ACEIs have higher levels of expression of ACE2, experimental and clinical studies have been performed to investigate the influence of ARBs or ACEIs on Covid19 [52] Moreover, spironolactone, a potassium-sparing diuretic 
drug antagonizing mineralocorticoid receptors, display favorable effects on ACE2 expression, preventing acute lung injuries, also in Covid19 [53, 54]. Therefore, several prospective randomized control trials investigating the protective effect of renin-angiotensin-aldosterone systemblockers in Covid19 are currently ongoing (REGISTRATION: URL: https://www.clinicaltrials.gov; Unique identifier: eg, NCT04493359, NCT04394117, NCT04591210, NCT04312009, NCT04351581, NCT04366050; and DRKS00021732, NCT04345887).

To date, the main advantage of drug repurposing lies in its economy. Indeed, the approaches used are faster and less expensive than the design and development of a drug from scratch. However, there are not many centers that can afford to perform a reliable computational approach and many of the drugs that have so far been identified with these approaches have not given the desired results.

Therefore, if on the one hand the strategy of using drugs already placed on the market to save time and resources appears fascinating, on the other hand it seems more likely that on a global level it will not be sufficient to change the fate of Covid19.

\section{Funding None.}

\section{Declarations}

Conflicts of interest Nothing to declare.

\section{References}

1. 2021. https://www.who.int/emergencies/diseases/novel-coronavirus-2019. Accessed 20 Aug 2021.

2. Savoia C, Volpe M, Kreutz R. Hypertension, a Moving Target in COVID-19: current views and perspectives. Circ Res. 2021;128:1062-79.

3. Hu B, Huang S, Yin L. The cytokine storm and COVID-19. J Med Virol. 2021;93:250-6.

4. Sertkaya A, Birkenbach A, Berlind A, Eyraud J. Examination of clinical trial costs and barriers for drug development. In: US Department of Health and Human Services, office of the assistant secretary for planning and evaluation report. 2014;1:1-92.

5. Yeu Y, Yoon Y, Park S. Protein localization vector propagation: a method for improving the accuracy of drug repositioning. Mol Biosyst. 2015;11:2096-102.

6. 2021. https://ourworldindata.org/covid-vaccinations. Accessed 20 Aug 2021.

7. Mahase E. Covid-19: what treatments are being investigated? BMJ. 2020;368:m1252.

8. The RECOVERY Collaborative Group. Dexamethasone in hospitalized patients with Covid-19. N Engl J Med. 2021;384:693-704.

9. Huang F, Zhang C, Liu Q, Zhao Y, Zhang Y, Qin Y, et al. Identification of amitriptyline $\mathrm{HCl}$, flavin adenine dinucleotide, azacitidine and calcitriol as repurposing drugs for influenza A H5N1 virus-induced lung injury. PLoS Pathog. 2020;16:e1008341.
10. Paolini GV, Shapland RH, van Hoorn WP, Mason JS, Hopkins AL. Global mapping of pharmacological space. Nat Biotechnol. 2006;24:805-15.

11. Koch U, Hamacher M, Nussbaumer P. Cheminformatics at the interface of medicinal chemistry and proteomics. Biochim Biophys Acta. 2014;1844:156-61.

12. Hodos RA, Kidd BA, Shameer K, Readhead BP, Dudley JT. In silico methods for drug repurposing and pharmacology. Wiley Interdiscip Rev Syst Biol Med. 2016;8:186-210.

13. Piro RM. Network medicine: linking disorders. Hum Genet. 2012;131:1811-20

14. Singh TU, Parida S, Lingaraju MC, Kesavan M, Kumar D, Singh RK. Drug repurposing approach to fight COVID-19. Pharmacol Rep. 2020;72(6):1479-508.

15. Solidarity' clinical trial for COVID-19 treatment. 2021. www. who.int. Accessed 20 Aug 2021.

16. Abel R, Paredes Ramos M, Chen Q, Pérez-Sánchez H, Coluzzi F, Rocco M, Marchetti P, Mura C, Simmaco M, Bourne PE, Preissner R, Banerjee P. Computational prediction of potential inhibitors of the main protease of SARS-CoV-2. Front Chem. 2020;8:590263.

17. RECOVERY Investigators. Dexamethasone in hospitalized patients with COVID-19- preliminary report. N Engl J Med 2021;384:693-704.

18. ACTT-1 Study Group Members. Remdesivir for the treatment of COVID-19-final report. N Engl J Med. 2020;383:1813-1826.

19. Kalil AC et al. ACTT-2 Study Group Members. Baricitinib plus remdesivir for hospitalized adults with COVID-19. N Engl J Med. 2021;384(9):795-80.

20. Nelson MR, Tipney H, Painter JL, Shen J, Nicoletti P, Shen Y, Floratos A, Sham PC, Li MJ, Wang J, Cardon LR, Whittaker JC, Sanseau P. The support of human genetic evidence for approved drug indications. Nat Genet. 2015;47(8):856-60.

21. VA Million Veteran Program COVID-19 Science Initiative. Actionable druggable genome-wide Mendelian randomization identifies repurposing opportunities for COVID-19. Nat Med. 2021;27:668-76.

22. Metushi IG, Wriston A, Banerjee P, Gohlke BO, English AM, Lucas A, et al. Acyclovir has low but detectable influence on HLA-B57:01 specificity without inducing hypersensitivity. PLoS ONE. 2015;10:e0124878.

23. Fiscon G, Conte F, Farina L, Paci P. SAveRUNNER: a network-based algorithm for drug repurposing and its application to COVID-19. PLoS Comput Biol. 2021;17:e1008686.

24. Fehr AR, Perlman S. Coronaviruses: an overview of their replication and pathogenesis. Coronaviruses. 2015;1282:1-23.

25. Gordon DE, et al. A sars-cov-2 protein interaction map reveals targets for drug repurposing. Nature. 2020;583:459-68.

26. Zhou Y, Hou Y, Shen J, Huang Y, Martin W, Cheng F. Network-based drug repurposing for novel coronavirus 2019-nCoV/ SARS-CoV-2. Cell Discov. 2020;2020:6.

27. Bianconi V, Violi F, Fallarino F, Pignatelli P, Sahebkar A, Pirro $M$. Is acetylsalicylic acid a safe and potentially useful choice for adult patients with COVID-19? Drugs. 2020;23:1-14.

28. Bikdeli B, Madhavan MV, Jimenez D, Chuich T, Dreyfus I, Driggin E, et al. COVID-19 and thrombotic or thromboembolic disease: implications for prevention, antithrombotic therapy, and follow-up: JACC state-of-the-art review. J Am Coll Cardiol. 2020;75:2950-73.

29. Inciardi RM, Lupi L, Zaccone G, Italia L, Raffo M, Tomasoni D, et al. Cardiac involvement in a patient with coronavirus disease 2019 (COVID-19). JAMA Cardiol. 2020;5:1-6.

30. Guo T, Fan Y, Chen M, Wu X, Zhang L, He T, et al. Cardiovascular implications of fatal outcomes of patients with coronavirus disease 2019 (COVID-19). JAMA Cardiol. 2020;5:1-8. 
31. Madjid M, Safavi-Naeini P, Solomon SD, Vardeny O. Potential effects of coronaviruses on the cardiovascular system: a review. JAMA Cardiol. 2020;5:831-40.

32. Volpe M, Battistoni A; board of the Italian Society of Cardiovascular Prevention, Bellotti P, Bellone S, Bertolotti M, Biffi A, Consoli A, Corsini A, Desideri G, Ferri C, Modena MG, Nati G, Pirro M, Rubattu S, Tocci G, Trimarco B, Volpe R, de Kreutzenberg SV. Recommendations for cardiovascular prevention during the Sars-Cov-2 pandemic: an executive document by the board of the Italian society of cardiovascular prevention. High Blood Press Cardiovasc Prev. 2020;27:373-7.

33. Sadowitz B, Maier KG, Gahtan V. Basic science review: statin therapy-part I: the pleiotropic effects of statins in cardiovascular disease. Vasc Endovasc Surg. 2010;44(4):241-51.

34. Zeiser R, Maas K, Youssef S, et al. Regulation of different inflammatory diseases by impacting the mevalonate pathway. Immunology. 2009;127(1):18-25.

35. Gordon D. Statins may be a key therapeutic for Covid-19. Med Hypotheses. 2020;144:110001.

36. Mehrbod P, Omar AR, Hair-Bejo M, Haghani A, Ideris A. Mechanisms of action and efficacy of statins against influenza. Biomed Res Int. 2014;2014:872370.

37. Gorabi AM, Kiaie N, Bianconi V, Jamialahmadi T, Al-Rasadi K, Johnston TP, et al. Antiviral effects of statins. Prog Lipid Res. 2020;2020:101054.

38. Tikoo K, Patel G, Kumar S, Karpe PA, Sanghavi M, Malek V, et al. Tissue specific up regulation of ACE2 in rabbit model of atherosclerosis by atorvastatin: role of epigenetic histone modifications. Biochem Pharmacol. 2015;93:343-51.

39. Wösten-van Asperen RM, Bos AP, Bem RA, Dierdorp BS, Dekker $\mathrm{T}$, van Goor $\mathrm{H}$, et al. Imbalance between pulmonary angiotensinconverting enzyme and angiotensinconverting enzyme 2 activity in acute respiratory distress syndrome. Pediatr Crit Care Med. $2013 ; 14: 438-41$

40. Zhang XJ, et al. In-hospital use of statins is associated with a reduced risk of mortality among individuals with COVID-19. Cell Metab Cell Metabolism. 2020;32:176-87.

41. Ganjali S, Bianconi V, Penson PE, Pirro M, Banach M, Watts GF, Sahebkar A. Commentary: Statins, COVID-19, and coronary artery disease: killing two birds with one stone. Metabolism Clin Exp. 2020;113:154375. https://doi.org/10.1016/j.metabol.2020. 154375.

42. CORONADO Investigators. Routine use of statins and increased COVID-19 related mortality in inpatients with type 2 diabetes: results from the CORONADO study. Diabetes Metab. 2021:47:101202.

43. André JS. Statins and clinical outcomes with COVID-19: meta-analyses of observational studies. Diabetes Metab. 2020;47(6): 101220

44. Onorato D, Pucci M, Carpene G, Henry BM, Sanchis-Gomar F, Lippi G. Protective effects of statins administration in european and north american patients infected with COVID-19: a metaanalysis. Semin Thromb Hemost. 2021;47:392-9.

45. McCarthy CP, Murphy S, Jones-O'Connor M, et al. Early clinical and sociodemographic experience with patients hospitalized with COVID-19 at a large American healthcare system. EClinicalMedicine. 2020;26:100504.

46. Tomizawa A, Hattori Y, Inoue T, Hattori S, Kasai K. Fenofibrate suppresses microvascular inflammation and apoptosis through adenosine monophosphate-activated protein kinase activation. Metabolism. 2011;60:513-22.

47. El-Arabey AA. Update on off label use of metformin for obesity. Prim Care Diabetes. 2018;12:3.

48. Luo $\mathrm{P}$, et al. Metformin treatment was associated with decreased mortality in COVID-19 patients with diabetes in a retrospective analysis. Am J Trop Med Hyg. 2020;103:69-72.

49. Hariyanto TI, Kurniawan A. Metformin use is associated with reduced mortality rate from coronavirus disease 2019 (COVID19) infection. Obes Med. 2020;2020:5.

50. Lukito AA, Pranata R, Henrina J, Lim MA, Lawrensia S, Suastika $\mathrm{K}$. The effect of metformin consumption on mortality in hospitalized COVID-19 patients: a systematic review and meta-analysis. Diabetes Metab Syndr. 2020;14:2177-83.

51. Bardaweel SK, Hajjo R, Sabbah DA. Sitagliptin: a potential drug for the treatment of COVID-19? Acta Pharm. 2021;71:175-84.

52. Volpe M, Battistoni A. Systematic review of the role of reninangiotensin system inhibitors in late studies on Covid-19: a new challenge overcome? Int J Cardiol. 2020;321:150-4.

53. Cadegiani FA, Goren A, Wambier CG. Spironolactone may provide protection from SARS-CoV-2: targeting androgens, angiotensin converting enzyme 2 (ACE2), and renin-angiotensin-aldosterone system (RAAS). Med Hypotheses. 2020;2020:143.

54. Cadegiani FA, Wambier CG, Goren A. Spironolactone: an antiandrogenic and anti-hypertensive drug with strong potential to prevent Covid-19 induced acute respiratory distress syndrome (ARDS)". Front Med. 2020;7:453. 